\title{
Gene expression profile analysis of ventilator-associated pneumonia
}

\author{
XIAOLI XU ${ }^{1}$, BO YUAN ${ }^{2}$, QUAN LIANG ${ }^{2}$, HUIMIN HUANG ${ }^{1}$, XIANGYI YIN ${ }^{1}$, \\ XIAOYUE SHENG ${ }^{1}$, NIUYAN NIE ${ }^{1}$ and HONGMEI FANG ${ }^{1}$ \\ Departments of ${ }^{1}$ Infection Management and ${ }^{2}$ Medical Administration, Jinling Hospital, \\ Medical School of Nanjing University, Nanjing, Jiangsu 210002, P.R. China
}

Received November 29, 2014; Accepted August 18, 2015

DOI: $10.3892 / \mathrm{mmr} .2015 .4389$

\begin{abstract}
Based on the gene expression profile of patients with ventilator-associated pneumonia (VAP) and patients not affected by the disease, the present study aimed to enhance the current understanding of VAP development using bioinformatics methods. The expression profile GSE30385 was downloaded from the Gene Expression Omnibus database. The Linear Models for Microarray Data package in R language was used to screen and identify differentially expressed genes (DEGs), which were grouped as up- and down-regulated genes. The up- and downregulated genes were functionally enriched using the Database for Annotation, Visualization and Integrated Discovery system and then annotated according to TRANSFAC, Tumor Suppressor Gene and Tumor Associated Gene databases. Subsequently, the protein-protein interaction (PPI) network was constructed, followed by module analysis using CFinder software. A total of 69 DEGs, including 33 upand 36 downregulated genes were screened out in patients with VAP. Upregulated genes were mainly enriched in functions and pathways associated with the immune response (including the genes ELANE and $L T F$ ) and the mitogen-activated protein kinase (MAPK) signaling pathway (including MAPK14). The PPI network comprised 64 PPI pairs and 44 nodes. The top two modules were enriched in different pathways, including the MAPK signaling pathway. Genes including ELANE, LTF and $M A P K 14$ may have important roles in the development
\end{abstract}

Correspondence to: Dr Xiaoli Xu, Department of Infection Management, Jinling Hospital, Medical School of Nanjing University, 305 East Zhongshan Road, Nanjing, Jiangsu 210002, P.R. China

E-mail: xiaolixuxxjd@163.com

Dr Bo Yuan, Department of Medical Administration, Jinling Hospital, Medical School of Nanjing University, 305 East Zhongshan Road, Nanjing, Jiangsu 210002, P.R. China

E-mail: boyuanyhdh@163.com

Key words: ventilator-associated pneumonia, differentially expressed genes, function enrichment, protein-protein interaction network, module analysis of VAP via altering the immune response and the MAPK signaling pathway.

\section{Introduction}

Ventilator-associated pneumonia (VAP), the most common type of hospital-acquired pneumonia (HAP), is defined as bacterial pneumonia that develops in patients who have received mechanical ventilation for $>48 \mathrm{~h}$ (1). VAP occurs in 6-52\% of mechanically ventilated patients and in certain specific settings, the incidence is up to $76 \%$ (2). The incidence of VAP varies depending on the type of population studied, the intensity of preventive measures implemented and the presence or absence of risk factors. With an estimated attributable mortality of $9 \%$, VAP usually increases the time of mechanical ventilation and time spent in the intensive care unit (ICU) by $>10$ days and its treatment accounts for at least $50 \%$ of antibiotics prescribed in ICUs, producing a huge cost (3). The expected burden of VAP in the coming years may increase with the increasing age of the population, intensification of care and the growing prevalence of severe underlying diseases in ICU patients (4). As the diagnosis as well as the treatment of VAP remain challenging, studies are required to gain a comprehensive understanding of VAP.

A number of previous studies have shown that up- or downregulation of the expression of certain genes is closely associated with the development of infection. For instance, a study on the polymorphism of tumor necrosis factor (TNF)- $\alpha$ reported that overexpression of TNF- $\alpha$ is associated with a 2.1-13-fold increase in the incidence of severe sepsis from all causes, including pneumonia (5). PI3, which encodes an elastase-specific inhibitor with antimicrobial peptide activity, has proven to promote early clearance of Pseudomonas aeruginosa by activating macrophages and recruiting neutrophils (6). PIK3R3, which encodes the protein of phosphoinositide 3-kinase regulatory sub-unit gamma, was shown to be predominately expressed in immune cells and to be involved in chemoattractant-induced cell migration (7). None of these genes alone, however, is sufficient enough to answer the fundamental question why certain patients develop VAP, while other similar patients do not.

As a genome-wide screening approach, microarray analysis may be effective for identifying novel genetic factors or gene expression profiles associated with the development of 
infection in VAP. With the goal to construct a model for the prediction of susceptibility to VAP in critically-injured trauma patients, Swanson et al (8) analyzed the gene expression profiles of patients who developed VAP and those who were never affected. By comparing the differentially expressed genes (DEGs) between the two groups, a logistic regression model was constructed, which comprised five genes (PIK3R3, ATP2A1, PI3, ADAM8 and HCN4) and was able to accurately categorize $95 \%$ of patients that developed VAP.

The present study identified DEGs between patients with VAP and those without VAP based on the expression profiling data provided by Swanson et al (8) and then performed functional annotation and enrichment analyses in order to elucidate the potential molecular mechanisms of VAP. In addition, a protein-protein interaction (PPI) network was constructed and the functional modules in this network were analyzed. The novel view on VAP provided by the present study indicates that VAP requires further study.

\section{Materials and methods}

Derivation of genetic data. The gene expression profile dataset GSE30385 deposited by Swanson et al (8) was downloaded from the Gene Expression Omnibus database (http://www.ncbi.nlm. nih.gov/geo), a public functional genomics data repository. The annotation platform was the GPL201 (HG-Focus) Affymetrix Human HG-Focus Target Array platform (Affymetrix, Inc., Santa Clara, CA, USA) updated on May 10th, 2013. A total of 20 samples from critically ill trauma patients were available, including 10 patients who developed ventilator-associated pneumonia (VAP group) and 10 who did not (NoVAP group).

Data processing. The Robust Multichip Average method of the R/Bioconductor Affy package (http://www.bioconductor.org/; Affymetrix, Inc.) was used to pre-process the downloaded raw data via background adjustment, quintile normalization and summarization (9). Using the R/Bioconductor software v 3.1 (http://www.bioconductor.org/), probe serial numbers in the matrix were transformed into gene names. As certain different probes can be mapped to the same gene, the average probe expression value was calculated as the final expression value of the corresponding gene.

Screening of DEGs. To screen DEGs between the VAP group and the NoVAP group, the Linear Models for Microarray Data (Limma) package in R/Bioconductor software 3.1 (http://www.bioconductor.org/packages/release/ bioc/html/limma.html) wasused(10). The Benjamini-Hochberg false discovery rate (FDR) was used to adjust the raw P-value in the multiple testing $(11,12)$. DEGs were screened with cutoff values of FDR $<0.05$ and $\mid \log$ (fold change) $\mid \geq 1.5(13,14)$. The eligible DEGs were grouped into upregulated and downregulated genes. To guarantee that the identified DEGs represent the two types of specimen, cluster analysis of DEGs was also performed using the Pheatmap package of $\mathrm{R}$ (http://cran.rproject.org/web/packages/pheatmap/index.html).

Functional annotation and pathway enrichment analysis. The Database for Annotation Visualization and Integrated Discovery (DAVID) provides a comprehensive set of functional annotation tools to understand the biological meaning of large lists of genes by using a hypergeometric distribution algorithm (15). To functionally annotate DEGs identified between the VAP group and the NoVAP group, DAVID was used to analyze the significantly enriched Gene Ontology (GO) terms (16) and Kyoto Encyclopedia of Genes and Genomes (KEGG) pathways (17) for upregulated and down-regulated genes, respectively (adjusted P-value <0.05).

Computational identification of transcription factors (TF), tumor suppressor genes (TSGs) and oncogenes. TRANSFAC version 7.0 (http://www.gene-regulation.com/pub/databases. html), which was initially a data collection, has evolved into the basis for a complex platform for the description and analysis of gene regulatory events and networks, including TFs as well as their DNA-binding sites and profiles (18). The TSGene database was designed by integrating TSGs with large-scale experimental evidence to offer a comprehensive resource for further investigation of TSGs and their molecular mechanisms in cancer (19). The tumor-associated gene (TAG) database was developed by integrating information from well-characterized oncogenes and tumor suppressor genes to facilitate cancer research (20). Based on these three databases, DEGs identified between the VAP group and the NoVAP group were analyzed for the screening of TFs, TSGs and TAGs.

Protein-protein interaction (PPI) network construction. The Search Tool for the Retrieval of Interacting Genes (STRING version 1.0; http://string-db.org/) is an online database which includes experimental as well as predicted interaction information and comprises $>1,100$ completely sequenced organisms (21). The DEGs identified above were directly mapped to the STRING database for acquiring significant PPI pairs from a range of sources, including data from experimental studies and data retrieved by text mining and homology searches. PPI pairs with the combined score of $\geq 0.4$ were retained for the construction of the PPI network.

Functional module analysis. CFinder (http://www.cfinder. org/) is a fast program for locating and visualizing overlapping, densely interconnected groups of nodes (defined as modules) in undirected graphs, and allows for easy navigation between the original graph and the network of these groups (22). Using the default parameter of $k=3$, modules were screened from the PPI network constructed as described above using CFinder. The top two sub-modules identified by modularity analysis were then selected for later functional enrichment by DAVID (FDR<0.05).

\section{Results}

Identification of DEGs. With a FDR $<0.05$ and $\mid \log$ (fold change) $\geq 1.5,69$ DEGs between the VAP group and the NoVAP group were obtained. Of these DEGs, $33(47.83 \%)$ were upregulated and $36(52.17 \%)$ were downregulated in the VAP group compared with the NoVAP group. The results of the clustering analysis of the DEGs are shown in Fig. 1.

Functional annotation and pathway enrichment analysis. To identify the functions of these DEGs, all of the up- and downregulated genes were mapped to terms of the GO data- 

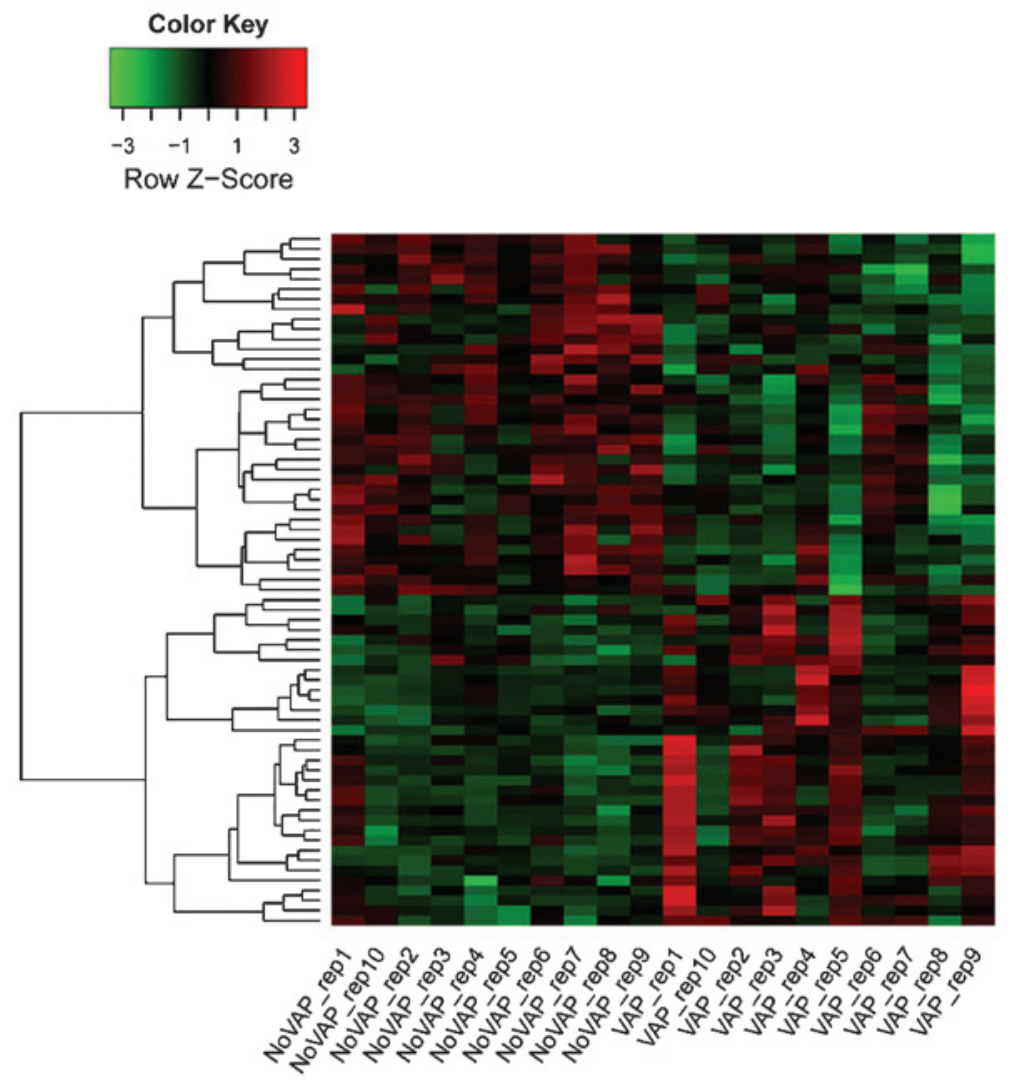

Figure 1. Dendrogram of differentially expressed genes identified by cluster analysis.

base, which consists of three ontologies, namely biological processes (BP), cellular components (CC) and molecular function (MF). In the three categories of the $\mathrm{GO}$ terms, 'immune system processes', 'immune response', 'kinase activity' were dominant for the upregulated genes, while 'response to stress', 'programmed cell death' and 'peptidase inhibitor activity' were dominant among the downregulated genes (Table I).

KEGG was used to further identify the altered biological functions arising from these DEGs. The upregulated genes were mainly enriched in ten pathways, including the neurotrophin signaling pathway, the mitogen-activated protein kinase (MAPK) signaling pathway and the nucleotide-binding oligomerization domain (NOD)-like receptor signaling pathway, while the downregulated genes were enriched in nine pathways, which included complement and coagulation cascades as well as pathways in cancer and ribosomes (Table II).

Identification of TFs, TSGs and oncogenes. According to the TRANSFAC, TSGene and TAG databases, a total of four TFs, two oncogenes, four TSGs and two other TAGs were further screened from the DEGs preliminarily identified between the VAP group and the NoVAP group (Table III).

PPI construction. The PPI network contained 64 PPI pairs and 44 nodes (20 upregulated genes and 24 downregulated genes in the VAP group) (Fig. 2). The five node proteins with a connection degree of $>5$ were ELANE (connection degree, 10), PTGS2 (connection degree, 9), MAPK14 (connection degree, 8), LTF (connection degree, 7) and MMP8 (connection degree, 6).

Functional module analysis. High aggregation, which reflects the high modularization of a gene network, is an important characteristic of biological networks. To distinguish the modules with specific functions and different sizes, the constructed network was usually divided into relatively independent sub-modules prior to analysis. By using CFinder, three models were obtained using the default parameter of $k=3$ and the top two modules are shown in Fig. 3.

The significantly enriched GO terms and KEGG pathways for Module 1 and Module 2 are displayed in Tables IV and V, respectively. Genes in Module 1 were mainly classified into the GO terms of multi-organism processes, extracellular region and serine-type endopeptidase activity, while genes in Module 2 were mainly classified into the GO terms negative regulation of biological processes, nucleus and protein kinase activity. Genes in Module 2 were enriched in 12 pathways, including the MAPK, p53 and VEGF signaling pathways, while no significant pathway was identified for genes in Module 1 at $F D R<0.05$.

\section{Discussion}

Due to its high prevalence and mortality, there is an urgent requirement to investigate the pathogenic mechanism of VAP. The present study analyzed the gene expression profiles of patients who developed VAP and those who were not affected in order to identify possible functions and signaling pathways 
Table I. Classification of differentially expressed genes in ventilator-associated pneumonia according to GO terms with $\mathrm{P}<0.05$.

\begin{tabular}{|c|c|c|c|c|}
\hline Gene category & GO ID & Function & Count & P-value \\
\hline \multicolumn{5}{|c|}{ Upregulated genes } \\
\hline \multirow[t]{5}{*}{$\mathrm{BP}$} & GO:0009617 & Response to bacterium & 9 & $6.66 \times 10^{-8}$ \\
\hline & GO:0006955 & Immune response & 14 & $2.12 \times 10^{-7}$ \\
\hline & GO:0051707 & Response to other organism & 9 & $5.28 \times 10^{-6}$ \\
\hline & GO:0009607 & Response to biotic stimulus & 9 & $7.59 \times 10^{-6}$ \\
\hline & GO:0002376 & Immune system process & 15 & $9.69 \times 10^{-6}$ \\
\hline \multirow[t]{5}{*}{$\mathrm{CC}$} & GO:0005576 & Extracellular region & 13 & 0.0001 \\
\hline & GO:0071682 & Endocytic vesicle lumen & 2 & 0.0004 \\
\hline & GO:0030139 & Endocytic vesicle & 4 & 0.0005 \\
\hline & GO:0005615 & Extracellular space & 6 & 0.0052 \\
\hline & GO:0045335 & Phagocytic vesicle & 2 & 0.0076 \\
\hline \multirow[t]{5}{*}{ MF } & GO:0004908 & Interleukin-1 receptor activity & 2 & $8.24 \times 10^{-5}$ \\
\hline & GO:0035251 & UDP-glucosyltransferase activity & 2 & 0.0001 \\
\hline & GO:0046527 & Glucosyltransferase activity & 2 & 0.0002 \\
\hline & GO:0004708 & Kinase activity & 2 & 0.0005 \\
\hline & GO:0004712 & Protein serine/threonine/tyrosine kinase activity & 2 & 0.0023 \\
\hline \multicolumn{5}{|c|}{ Downregulated genes } \\
\hline \multirow[t]{5}{*}{$\mathrm{BP}$} & GO:0050873 & Brown fat cell differentiation & 3 & $5.08 \times 10^{-5}$ \\
\hline & GO:0006950 & Response to stress & 18 & 0.0001 \\
\hline & GO:0050790 & Regulation of catalytic activity & 12 & 0.0001 \\
\hline & GO:0006915 & Apoptotic process & 12 & 0.0002 \\
\hline & GO:0012501 & Programmed cell death & 12 & 0.0002 \\
\hline \multirow[t]{5}{*}{$\mathrm{CC}$} & GO:0044445 & Cytosolic part & 4 & 0.0005 \\
\hline & GO:0022626 & Cytosolic ribosome & 3 & 0.0009 \\
\hline & GO:0044421 & Extracellular region part & 8 & 0.0019 \\
\hline & GO:0044391 & Ribosomal subunit & 3 & 0.0026 \\
\hline & GO:0005829 & Cytosol & 12 & 0.0036 \\
\hline \multirow[t]{5}{*}{ MF } & GO:0042277 & Peptide binding & 4 & 0.0004 \\
\hline & GO:0033218 & Amide binding & 4 & 0.0005 \\
\hline & GO:0004866 & Endopeptidase inhibitor activity & 4 & 0.0006 \\
\hline & GO:0061135 & Endopeptidase regulator activity & 4 & 0.0006 \\
\hline & GO:0030414 & Peptidase inhibitor activity & 4 & 0.0006 \\
\hline
\end{tabular}

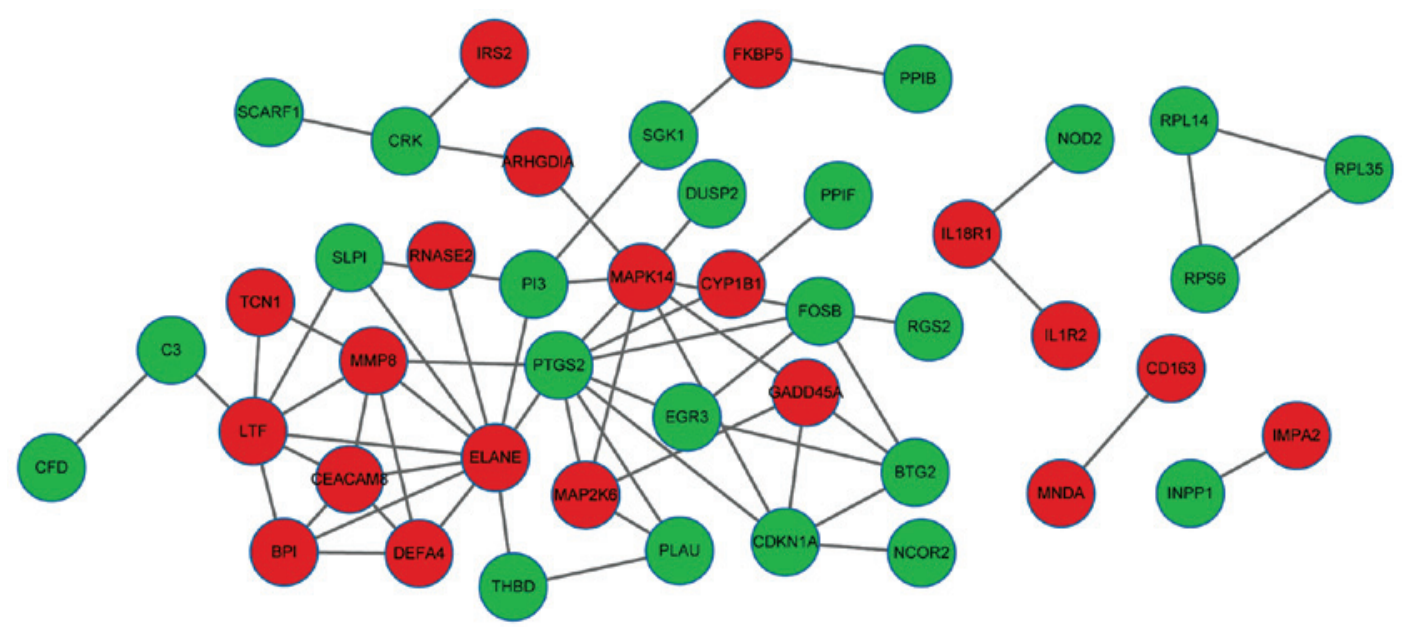

Figure 2. Protein-protein interaction networks of differentially expressed genes. 
Table II. Enrichment of KEGG pathways in ventilator-associated pneumonia with $\mathrm{P}<0.05$.

\begin{tabular}{|c|c|c|c|c|}
\hline Gene category & KEGG ID & Function & Count & P-value \\
\hline \multirow[t]{10}{*}{ Upregulated genes } & 4722 & Neurotrophin signaling pathway & 3 & 0.0064 \\
\hline & 4010 & Mitogen-activated protein kinase signaling & 4 & 0.0078 \\
\hline & 5014 & Amyotrophic lateral sclerosis & 2 & 0.0112 \\
\hline & 4621 & $\begin{array}{l}\text { Nucleotide-binding oligomerization domain } \\
\text { receptor signaling pathway }\end{array}$ & 2 & 0.0133 \\
\hline & 4664 & Fc epsilon RI signaling pathway & 2 & 0.0238 \\
\hline & 4640 & Hematopoietic cell lineage & 2 & 0.0291 \\
\hline & 4912 & Gonadotropin-releasing hormone signaling & 2 & 0.0375 \\
\hline & 4620 & Toll-like receptor signaling pathway & 2 & 0.0382 \\
\hline & 5146 & Amoebiasis & 2 & 0.0410 \\
\hline & 4060 & Cytokine-cytokine receptor interaction & 3 & 0.0449 \\
\hline \multirow[t]{9}{*}{ Downregulated genes } & 4610 & Complement and coagulation cascades & 4 & 0.0001 \\
\hline & 3010 & Ribosome & 3 & 0.0053 \\
\hline & 5150 & Staphylococcus aureus infection & 2 & 0.0192 \\
\hline & 5131 & Shigellosis & 2 & 0.0234 \\
\hline & 5140 & Leishmaniasis & 2 & 0.0318 \\
\hline & 5220 & Chronic myeloid leukemia & 2 & 0.0326 \\
\hline & 5200 & Pathways in cancer & 4 & 0.0358 \\
\hline & 5222 & Small cell lung cancer & 2 & 0.0431 \\
\hline & 4012 & ErbB signaling pathway & 2 & 0.0449 \\
\hline
\end{tabular}

KEGG, Kyoto Encyclopedia of Genes and Genomes.

Table III. Annotation for TFs, TSGs, oncogenes and other TAGs in ventilator-associated pneumonia.

\begin{tabular}{lcccc}
\hline Gene category & TFs & Oncogenes & TSGs & Other TAGs \\
\hline Upregulated genes & KLF7 & CD24 & LTF & NA \\
Downregulated genes & EGR3, FOSB, NCOR2 & CRK & BTG2, CDKN1A, THBD & RGS2, CTSZ
\end{tabular}

TF, transcription factor; TSG, tumor suppressor gene; TAG, tumor-associated gene.

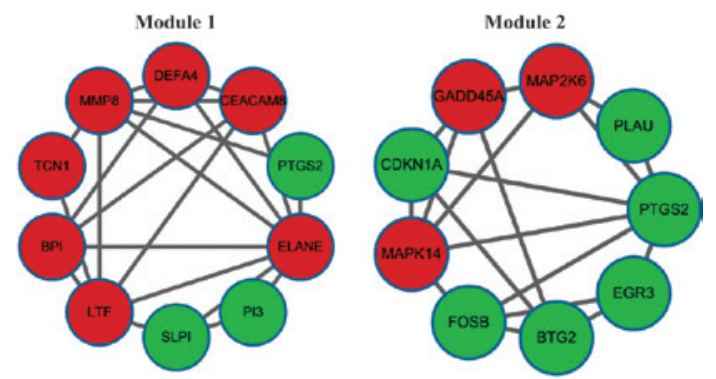

Figure 3. Top two modules in the protein-protein interaction network.

of DEGs between the two groups. After the construction of a PPI network, sub-modules in the network were mined and the functions of the top two modules were annotated.

In the present study, a total of 69 DEGs were identified between patients with VAP and those not affected. This result differs from that of Swanson et al (8), who provided the gene expression profile and identified 810 DEGs between the two groups. The inconsistency between the present study and that by Swanson et al (8) is likely to be attributed to the different statistical methods employed for detecting DEGs. Swanson et al (8) screened the DEGs using Partek software and the analysis of variance method, which calculates a $q$-value as described by Storey and Tibshirani (23), while the present study used the Limma package in R language, which is at present most commonly applied for identifying DEGs. To avoid false-positive results, relatively high cutoff values of FDR $<0.05$ and $\log$ (fold change) $\mid \geq 1.5$ were set, which ensured high accuracy of the results.

The upregulated genes in the VAP group identified in the present study were mainly enriched in the GO terms of immune system processes and immune responses, which have been verified to have a pivotal role in the development of VAP (24). The immune system has a central role in controlling the duration and amplitude of the inflammatory response (25). A previous study on sepsis revealed that the innate and adaptive immune response is considerably different between 
Table IV. GO annotation for genes in the top two modules.

\begin{tabular}{|c|c|c|c|c|}
\hline Category & GO ID & Function & Count & P-value \\
\hline \multicolumn{5}{|l|}{ Module 1} \\
\hline \multirow[t]{5}{*}{$\mathrm{BP}$} & GO:0050832 & Defense response to fungus & 3 & $3.87 \times 10^{-7}$ \\
\hline & GO:0009620 & Response to fungus & 3 & $1.42 \times 10^{-6}$ \\
\hline & GO:0009617 & Response to bacterium & 5 & $1.96 \times 10^{-6}$ \\
\hline & GO:0051704 & Multi-organism process & 7 & $5.46 \times 10^{-6}$ \\
\hline & GO:0051707 & Response to other organisms & 5 & $2.53 \times 10^{-5}$ \\
\hline \multirow[t]{5}{*}{$\mathrm{CC}$} & GO:0005576 & Extracellular region & 9 & $8.31 \times 10^{-8}$ \\
\hline & GO:0031012 & Extracellular matrix & 3 & 0.0016 \\
\hline & GO:0044421 & Extracellular region part & 4 & 0.0031 \\
\hline & GO:0030141 & Secretory granule & 2 & 0.0103 \\
\hline & GO:0005615 & Extracellular space & 3 & 0.0114 \\
\hline \multirow[t]{5}{*}{ MF } & GO:0004252 & Serine-type endopeptidase activity & 3 & $5.05 \times 10^{-5}$ \\
\hline & GO:0008236 & Serine-type peptidase activity & 3 & $7.44 \times 10^{-5}$ \\
\hline & GO:0017171 & Serine hydrolase activity & 3 & $7.70 \times 10^{-5}$ \\
\hline & GO:0004175 & Endopeptidase activity & 3 & 0.0007 \\
\hline & GO:0004867 & Serine-type endopeptidase inhibitor activity & 2 & 0.00103 \\
\hline \multicolumn{5}{|l|}{ Module 2} \\
\hline \multirow[t]{5}{*}{$\mathrm{BP}$} & GO:0071478 & Cellular response to radiation & 4 & $4.48 \times 10^{-7}$ \\
\hline & GO:2000379 & Positive regulation of reactive oxygen species metabolic process & 3 & $9.14 \times 10^{-7}$ \\
\hline & GO:0048519 & Negative regulation of biological processes & 9 & $1.27 \times 10^{-6}$ \\
\hline & GO:0009605 & Response to external stimuli & 7 & $1.63 \times 10^{-6}$ \\
\hline & GO:0071479 & Cellular response to ionizing radiation & 3 & $2.02 \times 10^{-6}$ \\
\hline \multirow[t]{2}{*}{$\mathrm{CC}$} & GO:0005634 & Nucleus & 7 & 0.0038 \\
\hline & GO:0005654 & Nucleoplasm & 3 & 0.0219 \\
\hline \multirow[t]{5}{*}{ MF } & GO:0004708 & Mitogen-activated protein kinase kinase activity & 2 & $3.65 \times 10^{-5}$ \\
\hline & GO:0004712 & Protein serine/threonine/tyrosine kinase activity & 2 & 0.00018 \\
\hline & GO:0004672 & Protein kinase activity & 3 & 0.0038 \\
\hline & GO:0016773 & Phosphotransferase activity, alcohol group as acceptor & 3 & 0.0064 \\
\hline & GO:0016301 & Kinase activity & 3 & 0.0079 \\
\hline
\end{tabular}

GO, gene ontology; BP, biological process; CC, cellular components; MF, molecular function.

Table V. KEGG pathway enrichment for genes in Module 2.

\begin{tabular}{|c|c|c|c|}
\hline KEGG ID & Function & Count & P-value \\
\hline 4380 & Osteoclast differentiation & 3 & 0.0005 \\
\hline 5014 & Amyotrophic lateral sclerosis & 2 & 0.0022 \\
\hline 4115 & P53 signaling pathway & 2 & 0.0035 \\
\hline 5140 & Leishmaniasis & 2 & 0.0040 \\
\hline 4370 & Vascular endothelial growth factor signaling pathway & 2 & 0.0044 \\
\hline 4010 & Mitogen-activated protein kinase signaling pathway & 3 & 0.0044 \\
\hline 4664 & Fc epsilon RI signaling pathway & 2 & 0.0048 \\
\hline 4912 & Gonadotropin-releasing hormone signaling pathway & 2 & 0.0077 \\
\hline 4620 & Toll-like receptor signaling pathway & 2 & 0.0078 \\
\hline 4110 & Cell cycle & 2 & 0.0114 \\
\hline 5145 & Toxoplasmosis & 2 & 0.0129 \\
\hline 5160 & Hepatitis C & 2 & 0.0132 \\
\hline
\end{tabular}

KEGG, Kyoto Encyclopedia of Genes and Genomes. 
patients with VAP and those without this type of nosocomial infection (26). A cohort study including 90 patients with VAP mainly caused by Gram-negative bacteria demonstrated an association between immune system disorders and mortality, as patients with early monocyte apoptosis of $>50 \%$ were less likely to succumb to sepsis compared with those exhibiting monocyte apoptosis of $1<50 \%$ (27). The present study revealed that GO:0002376 (immune system processes) and GO:0006955 (immune responses) were significantly abnormal in patients with VAP, and the two GO categories included the genes ELANE and LTF. ELANE is the gene encoding neutrophil elastase, a serine protease expressed in myelomonocytic cells and their precursors (28). Mutations in the ELANE gene are proven to be the most common genetic cause of congenital neutropenia, as ELANE mutations are associated with functional deficiencies in neutrophils and further contribute to the risk of infection $(29,30)$. In addition, lactoferrin, encoded by $L T F$, was suggested to affect the regulation of the inflammatory response by modulating cellular iron homeostasis (31). Furthermore, the present study showed that ELANE and LTF were the significant nodes in the resulting PPI network. It is therefore inferred that ELANE and LTF may be involved in the susceptibility of patients to VAP by affecting the immune response.

The present study also observed that the upregulated genes in patients with VAP were significantly enriched in the MAPK signaling pathway, which was also the most significant pathway enriched among genes in Module 2 mined from the PPI network. The MAPK signaling pathway is a three-tiered cascade that includes a MAP kinase kinase kinase (MAP3K), MAP kinase kinase (MAP2K) and MAPK, which regulate numerous cellular functions, including proliferation, differentiation, migration and apoptosis (32). The four major MAPKs are extracellular regulated kinases 1 and 2 (ERK1/2), c-Jun-N-terminal kinases, p38 and ERK5 (33). Functional analysis performed in the present study demonstrated that four DEGs (GADD45A, $M A P K 14, I L 1 R 2$ and MAP2K6) were significantly enriched in this pathway, among which MAPK14 is also one of the nodes with the highest connection degree in the PPI network. $M A P K 14$ encodes the MAPK14 protein that is also referred to as $\mathrm{p} 38 \alpha$ (34). p38 $\alpha$ is the first identified member of the p38 MAPK family and can be targeted by pyridinylimidazole drugs that inhibit the production of pro-inflammatory cytokines (35). Höcker et al (36) have demonstrated that following its activation by the GADD45B-MAP3K4 signaling complex, MAPK14 is directed to autophagosomes, where it impairs autophagosome-lysosome fusion and thus autophagy. It has been indicated that activation of autophagy in macrophages mediates the early lung inflammation during mechanical ventilation via NLRP3 inflammasome signaling (37). These findings combined with the results of the present study lead to the hypothesis that the p38 MAPK signaling pathway may be involved in the development of VAP and MAPK14 may be one of the key genes in this process.

In conclusion, the present study analyzed the gene expression profiles between patients with VAP and those not affected by this disease using a computational bioinformatics approach. Functional annotation of the DEGs into GO terms and KEGG pathways was performed, and a PPI network was constructed, followed by module mining. Genes including ELANE, LTF and MAPK14 may have important roles in the development of VAP via altering the immune response and the MAPK signaling pathway. The present study provided a novel, genetic perspective on VAP, which may aid in the development of strategies to prevent or treat VAP in patients in ICUs.

\section{Acknowledgements}

The present study was supported by the Nosocomial Infection Control Research Fund of The Chinese Preventative Association (grant no. ZHYG2014-0037) and the Jinling Hospital Research Fund of the Jinling Affiliated Hospital of Nanjing University Medical School (grant no. YYMS2014017).

\section{References}

1. Morehead RS and Pinto SJ: Ventilator-associated pneumonia. Arch Intern Med 160: 1926-1936, 2000.

2. Koenig SM and Truwit JD: Ventilator-associated pneumonia: Diagnosis, treatment and prevention. Clin Microbiol Rev 19: 637-657, 2006.

3. Kollef MH, Hamilton CW and Ernst FR: Economic impact of ventilator-associated pneumonia in a large matched cohort. Infect Control Hosp Epidemiol 33: 250-256, 2012.

4. Barbier F, Andremont A, Wolff $M$ and Bouadma L: Hospital-acquired pneumonia and ventilator-associated pneumonia: Recent advances in epidemiology and management. Curr Opin Pulm Med 19: 216-228, 2013.

5. O'Dwyer MJ, Mankan AK, Stordeur P, O'Connell B, Duggan E, White M, Kelleher DP, McManus R and Ryan T: The occurrence of severe sepsis and septic shock are related to distinct patterns of cytokine gene expression. Shock 26: 544-550, 2006.

6. Bridge DR, Novotny MJ, Moore ER and Olson JC: Role of host cell polarity and leading edge properties in Pseudomonas type III secretion. Microbiology 156: 356-373, 2010.

7. Ferrandi C, Ardissone V, Ferro P, Rückle T, Zaratin P, Ammannati E, Hauben E, Rommel C and Cirillo R: Phosphoinositide 3-kinase gamma inhibition plays a crucial role in early steps of inflammation by blocking neutrophil recruitment. J Pharmacol Exp Ther 322: 923-930, 2007.

8. Swanson JM, Wood GC, Xu L, Tang LE, Meibohm B, Homayouni R, Croce MA and Fabian TC: Developing a gene expression model for predicting ventilator-associated pneumonia in trauma patients: A pilot study. PLoS One 7: 15, 2012.

9. Ma J and Qin ZS: Different normalization strategies for microarray gene expression traits affect the heritability estimation. BMC Proc 1 (Suppl 1): S154, 2007.

10. Delhomme N, Padioleau I, Furlong EE and Steinmetz LM: EasyRNASeq: A bioconductor package for processing RNA-Seq data. Bioinformatics 28: 2532-2533, 2012.

11. Singh B, Ronghe AM, Chatterjee A, Bhat NK and Bhat HK: MicroRNA-93 regulates NRF2 expression and is associated with breast carcinogenesis. Carcinogenesis 34: 1165-1172, 2013.

12. Chand Y and Alam MA: Network biology approach for identifying key regulatory genes by expression based study of breast cancer. Bioinformation 8: 1132-1138, 2012.

13. Benjamini Y and Hochberg Y: Controlling the false discovery rate: A practical and powerful approach to multiple testing. J R Stat Soc B (Statistical Methodology) 57: 289-300, 1995.

14. Benjamini Y: Discovering the false discovery rate. J R Stat Soc B (Statistical Methodology) 72: 405-416, 2010.

15. Hu YH, Hu YL, Liu DW, Yu JX and Liu DM: Screening and bioinformatics analysis of differentially expressed genes in hyperplastic scar. Nan Fang Yi Ke Da Xue Xue Bao 34: 939-944, 2014 (In Chinese).

16. Ashburner M, Ball CA, Blake JA, Botstein D, Butler H, Cherry JM, Davis AP, Dolinski K, Dwight SS, Eppig JT, et al: Gene ontology: Tool for the unification of biology. The Gene Ontology Consortium. Nat Genet 25: 25-29, 2000.

17. Kanehisa M: The KEGG database. Novartis Found Symp 247: 91-101; discussion 101-103, 119-128, 244-252, 2002.

18. Wingender E: The TRANSFAC project as an example of framework technology that supports the analysis of genomic regulation. Brief Bioinform 9: 326-332, 2008. 
19. Zhao M, Sun J and Zhao Z: TSGene: A web resource for tumor suppressor genes. Nucleic Acids Res 41 (Database Issue): D970-D976, 2013.

20. Chen JS, Hung WS, Chan HH, Tsai SJ and Sun HS: In silico identification of oncogenic potential of fyn-related kinase in hepatocellular carcinoma. Bioinformatics 29: 420-427, 2013.

21. Franceschini A, Szklarczyk D, Frankild S, Kuhn M, Simonovic M, Roth A, Lin J, Minguez P, Bork P, von Mering C and Jensen LJ: STRING v9.1: Protein-protein interaction networks, with increased coverage and integration. Nucleic Acids Res 41 (Database Issue): D808-D815, 2013.

22. Adamcsek B, Palla G, Farkas IJ, Derényi I and Vicsek T: CFinder: Locating cliques and overlapping modules in biological networks. Bioinformatics 22: 1021-1023, 2006.

23. Storey JD and Tibshirani R: Statistical significance for genomewide studies. Proc Natl Acad Sci USA 100: 9440-9445, 2003.

24. Gogos C, Kotsaki A, Pelekanou A, Giannikopoulos G, Vaki I, Maravitsa P, Adamis S, Alexiou Z, Andrianopoulos G, Antonopoulou A, et al: Early alterations of the innate and adaptive immune statuses in sepsis according to the type of underlying infection. Crit Care 14: R96, 2010.

25. Surbatovic M, Jevdjic J, Veljovic M, Popovic N, Djordjevic D and Radakovic S: Immune response in severe infection: Could life-saving drugs be potentially harmful? Sci World J 2013: 961852, 2013.

26. Pelekanou A, Tsangaris I, Kotsaki A, Karagianni V, Giamarellou H, Armaganidis A and Giamarellos-Bourboulis EJ: Decrease of CD4-lymphocytes and apoptosis of CD14-monocytes are characteristic alterations in sepsis caused by ventilator-associated pneumonia: Results from an observational study. Crit Care 13: R172, 2009.

27. Giamarellos-Bourboulis EJ, Routsi C, Plachouras D, Markaki V, Raftogiannis M, Zervakis D, Koussoulas V, Orfanos S, Kotanidou A, Armaganidis A, et al: Early apoptosis of blood monocytes in the septic host: Is it a mechanism of protection in the event of septic shock? Crit Care 10: R76, 2006.
28. Germeshausen M, Deerberg S, Peter Y, Reimer C, Kratz CP and Ballmaier M: The spectrum of ELANE mutations and their implications in severe congenital and cyclic neutropenia. Hum Mutat 34: 905-914, 2013.

29. Shu Z, Li XH, Bai XM, Zhang ZY, Jiang LP, Tang XM and Zhao XD: Clinical characteristics of severe congenital neutropenia caused by novel ELANE gene mutations. Pediatr Infect Dis J 34: 203-207, 2015.

30. Horwitz MS, Corey SJ, Grimes HL and Tidwell T: ELANE mutations in cyclic and severe congenital neutropenia: Genetics and pathophysiology. Hematol Oncol Clin North Am 27: 19-41, 2013.

31. Frioni A, Conte MP, Cutone A, Longhi C, Musci G, di Patti MC, Natalizi T, Marazzato M, Lepanto MS, Puddu P, et al: Lactoferrin differently modulates the inflammatory response in epithelial models mimicking human inflammatory and infectious diseases. Biometals 27: 843-856, 2014.

32. Yang SH, Sharrocks AD and Whitmarsh AJ: MAP kinase signalling cascades and transcriptional regulation. Gene 513: $1-13,2013$

33. Slattery ML, Lundgreen A and Wolff RK: Dietary influence on MAPK-signaling pathways and risk of colon and rectal cancer. Nutr Cancer 65: 729-738, 2013.

34. Cuadrado A and Nebreda AR: Mechanisms and functions of p38 MAPK signalling. Biochem J 429: 403-417, 2010.

35. Yego EC and Dillman JF III: Cytokine regulation by MAPK activated kinase 2 in keratinocytes exposed to sulfur mustard. Toxicol In Vitro 27: 2067-2075, 2013.

36. Höcker R, Walker A and Schmitz I: Inhibition of autophagy through MAPK14-mediated phosphorylation of ATG5. Autophagy 9: 426-428, 2013.

37. Zhang Y, Liu G, Dull RO, Schwartz DE and Hu G: Autophagy in pulmonary macrophages mediates lung inflammatory injury via NLRP3 inflammasome activation during mechanical ventilation. Am J Physiol Lung Cell Mol Physiol 307: L173-L185, 2014. 